\section{(2) OPEN ACCESS}

Handling editor Josef $S$

Smolen

- Additional material is published online only. To view please visit the journal online (http://dx.doi.org/10.1136/ annrheumdis-2020-219209)

For numbered affiliations see end of article.

\section{Correspondence to}

Dr Chikashi Terao, Laboratory for Statistical and Translational Genetics Analysis, RIKEN Center for Integrative Medical Sciences, Kanagawa, Japan;

chikashi.terao@riken.jp,

Professor Yong Cui, Department of Dermatology, China-Japan Friendship Hospital, Beijing,

China;

wuhucuiyong@vip.163.com and Professor Sang-Cheol Bae, Rheumatology, Hanyang University Hospital for Rheumatic Diseases, Seoul, The Republic of Korea; scbae@hanyang.ac.kr

XY, KKim and HS contributed equally.

Received 30 September 2020 Revised 4 November 2020 Accepted 11 November 2020 Published Online First 3 December 2020

\section{Check for updates}

(c) Author(s) (or their employer(s)) 2021. Re-use permitted under CC BY-NC. No commercial re-use. See rights and permissions. Published by BMJ.

To cite: Yin $X_{\text {, Kim } \mathrm{K},}$

Suetsugu $\mathrm{H}$, et al.

Ann Rheum Dis

2021;80:632-640.

\title{
Meta-analysis of 208370 East Asians identifies 113 susceptibility loci for systemic lupus erythematosus
}

Xianyong Yin (1) , 1,2,3,4,5,6 Kwangwoo Kim (1) , ${ }^{7}$ Hiroyuki Suetsugu, 8,9,10

So-Young Bang, ${ }^{11,12}$ Leilei Wen, ${ }^{1,2,3}$ Masaru Koido, ${ }^{9,13}$ Eunji Ha, ${ }^{7}$ Lu Liu, 1,2,3

Yuma Sakamoto, ${ }^{14}$ Sungsin Jo, ${ }^{12}$ Rui-Xue Leng (1) , ${ }^{15}$ Nao Otomo, $8,9,16$

Viktoryia Laurynenka, ${ }^{17}$ Young-Chang Kwon, ${ }^{12}$ Yujun Sheng, ${ }_{1}^{1,2,3}$

Nobuhiko Sugano (D) , ${ }^{18}$ Mi Yeong Hwang, ${ }^{19}$ Weiran Li, ${ }^{1,2,3}$ Masaya Mukai, ${ }^{20}$ Kyungheon Yoon, ${ }^{19}$ Minglong Cai, ${ }^{1,2,3}$ Kazuyoshi Ishigaki, ${ }^{9,21,22,23}$ Won Tae Chung, ${ }^{24}$ He Huang, ${ }^{1,2,3}$ Daisuke Takahashi, ${ }^{25}$ Shin-Seok Lee, ${ }^{26}$ Mengwei Wang, 1,2,3 Kohei Karino, ${ }^{27}$ Seung-Cheol Shim, ${ }^{28}$ Xiaodong Zheng, ${ }^{1,2,3}$ Tomoya Miyamura, ${ }^{29}$ Young Mo Kang, ${ }^{30}$ Dongqing Ye (D) , ${ }^{15}$ Junichi Nakamura, ${ }^{31}$ Chang-Hee Suh, ${ }^{32}$ Yuanjia Tang, ${ }^{33}$ Goro Motomura, ${ }^{10}$ Yong-Beom Park, ${ }^{34}$ Huihua Ding, ${ }^{33}$ Takeshi Kuroda, ${ }^{35}$ Jung-Yoon Choe, ${ }^{36}$ Chengxu Li, ${ }^{5}$ Hiroaki Niiro, ${ }^{37}$ Youngho Park, ${ }^{12}$ Changbing Shen, ${ }^{38,39}$ Takeshi Miyamoto, ${ }^{40}$ Ga-Young Ahn, ${ }^{11}$ Wenmin Fei, ${ }^{5}$ Tsutomu Takeuchi, ${ }^{41}$ Jung-Min Shin, ${ }^{11}$ Keke Li, ${ }^{5}$ Yasushi Kawaguchi, ${ }^{42}$ Yeon-Kyung Lee, ${ }^{11}$ Yongfei Wang (1) " ${ }^{43}$ Koichi Amano, ${ }^{44}$ Dae Jin Park, ${ }^{11}$ Wanling Yang (D) , ${ }^{43}$ Yoshifumi Tada, ${ }^{45}$ Ken Yamaji, ${ }^{46}$ Masato Shimizu, ${ }^{47}$ Takashi Atsumi, ${ }^{48}$ Akari Suzuki, ${ }^{49}$ Takayuki Sumida, ${ }^{50}$ Yukinori Okada (D) , 51,52 Koichi Matsuda, ${ }^{53,54}$ Keitaro Matsuo, ${ }^{55,56}$ Yuta Kochi, ${ }^{57}$ Japanese Research Committee on Idiopathic Osteonecrosis of the Femoral Head, Leah C Kottyan (iD) , 17,58 Matthew T Weirauch, ${ }^{17,58}$ Sreeja Parameswaran, ${ }^{17}$ Shruti Eswar, ${ }^{17}$ Hanan Salim, ${ }^{17}$ Xiaoting Chen, ${ }^{17}$ Kazuhiko Yamamoto (D) , ${ }^{49}$ John B Harley, ${ }^{17,58,59}$ Koichiro Ohmura, ${ }^{60}$ Tae-Hwan Kim (1) ${ }^{11,12}$ Sen Yang, ${ }^{1,2,3}$ Takuaki Yamamoto, ${ }^{61}$ Bong-Jo Kim ${ }^{19}$ Nan Shen (D) , ${ }^{17,33,62}$ Shiro Ikegawa, ${ }^{8}$ Hye-Soon Lee, ${ }^{11,12}$ Xuejun Zhang, ${ }^{1,2,3,63}$ Chikashi Terao (D) , 9,64,65 Yong Cui, ${ }^{5}$ Sang-Cheol Bae (D) ${ }^{11,12}$

\section{ABSTRACT}

Objective Systemic lupus erythematosus (SLE), an autoimmune disorder, has been associated with nearly 100 susceptibility loci. Nevertheless, these loci only partially explain SLE heritability and their putative causal variants are rarely prioritised, which make challenging to elucidate disease biology. To detect new SLE loci and causal variants, we performed the largest genome-wide meta-analysis for SLE in East Asian populations.

Methods We newly genotyped 10029 SLE cases and 180167 controls and subsequently meta-analysed them jointly with 3348 SLE cases and 14826 controls from published studies in East Asians. We further applied a Bayesian statistical approach to localise the putative causal variants for SLE associations.

Results We identified 113 genetic regions including 46 novel loci at genome-wide significance $\left(p<5 \times 10^{-8}\right)$. Conditional analysis detected 233 association signals within these loci, which suggest widespread allelic heterogeneity. We detected genome-wide associations at six new missense variants. Bayesian statistical finemapping analysis prioritised the putative causal variants to a small set of variants ( $95 \%$ credible set size $\leq 10)$ for 28 association signals. We identified 110 putative

\section{Key messages}

What is already known about this subject?

- Genome-wide association studies have identified nearly 100 susceptibility loci for systemic lupus erythematosus (SLE) risk.

- The known SLE loci explain partially the disease heritability.

What does this study add?

- This study identified 113 genomic regions including 46 novel loci for SLE risk.

- The study prioritised 110 putative causal variants including 10 putative causal variants with high confidence (posterior probability $\geq 0.8$ ).

How might this impact on clinical practice or future developments?

- These findings revealed new genetic basis for SLE and generated molecular mechanisms hypotheses for further investigations. 
causal variants with posterior probabilities $\geq 0.1$ for 57 SLE loci, among which we prioritised 10 most likely putative causal variants (posterior probability $\geq 0.8$ ). Linkage disequilibrium score regression detected genetic correlations for SLE with albumin/globulin ratio $\left(r_{g}=-0.242\right)$ and non-albumin protein $\left(r_{q}=0.238\right)$.

Conclusion This study reiterates the power of large-scale genomewide meta-analysis for novel genetic discovery. These findings shed light on genetic and biological understandings of SLE.

\section{INTRODUCTION}

Systemic lupus erythematosus (SLE) is an autoimmune disorder characterised by the production of autoantibodies that damage multiple organs. ${ }^{1}$ Considerable genetic predisposition contributes to SLE aetiology. ${ }^{2}$ To date, nearly 100 susceptibility loci have been identified for SLE, mainly through genome-wide association studies (GWASs). ${ }^{3-8}$ However, these loci collectively only explain $\sim 30 \%$ of SLE heritability ${ }^{9}$ and their biology, in terms of causal variants, effector genes and cell types and pathological pathways that mediate genetic effects, has not yet been fully characterised. ${ }^{10}$

Genome-wide association meta-analyses have been performed to uncover new genetic associations for SLE in Asians, ${ }^{11}$ Europeans $^{12}$ and trans-ancestral populations. ${ }^{9}$ However, the study sample sizes were relatively modest, which limits their ability for genetic discovery. GWASs have successfully linked genetic variants with human common diseases and traits. ${ }^{13}$ Nonetheless, only $\sim 8 \%$ of GWAS participants are East Asians. ${ }^{14}$ East Asians have a unique population genetic history and may have ethnicity-specific genetic architecture involved in the development of disease and manifestations. For example, SLE has a remarkably higher prevalence and younger age of onset in Asians. ${ }^{15} 16$ Genetic heterogeneity may explain, at least partly, the phenotypic diversity of SLE between East Asians and Europeans. ${ }^{9}$ Hence, large-scale East Asian investigations may provide an opportunity to identify unique genetic associations even for the same diseases and traits that have already been well studied in Europeans. ${ }^{17}$

\section{METHODS}

\section{Study participants}

We recruited a total of 10029 SLE cases and 180167 healthy controls in three independent case-control cohorts from mainland China, Korea and Japan. We analysed additionally 3348 SLE cases and 14826 controls that were published in our previous East Asian SLE GWASs ${ }^{4-9}$ to increase statistical power. All the cases fulfilled the revised American College of Rheumatology SLE classification criteria or were diagnosed by collagen disease physicians (online supplemental table 1). Each participant provided written informed consent.

\section{Genome-wide association analyses}

We newly genotyped 10029 SLE cases and 180167 controls, and revisited raw genome-wide genotype data in 3348 SLE cases and 14826 controls from the five published studies. ${ }^{4-9}$ Quality controls were conducted for each of the eight data sets. Genotype imputation was accomplished using reference panels from the 1000 Genomes Project (1KGP) phase $3 \mathrm{v} 5^{18}$ and populationspecific reference panels ${ }^{19}$ in IMPUTE2/4 ${ }^{20} 21$ or MINIMAC4. ${ }^{22}$

We tested association between SLE risk and genotype dosages in each data set using a logistic regression or linear mixed model in PLINK, ${ }^{23}$ SNPTEST ${ }^{24}$ or EPACTS (https:// genome.sph.umich.edu/wiki/EPACTS) (online supplemental table 1). Within each data set, we filtered out association results based on imputation quality (IMPUTE info or MINIMAC $\mathrm{r}^{2} \leq 0.3$ ), minor allele frequency (MAF) $\leq 0.5 \%$ or Hardy-Weinberg equilibrium test $\mathrm{p}<1.0 \times 10^{-6}$ in controls. For each cohort, the association analysis for the $\mathrm{X}$ chromosome was conducted separately by sex and then meta-analysed across both men and women. For data sets analysed using a linear mixed model (online supplemental table 1), allelic effects and standard errors were converted to a $\log$-odds scale to correct for case-control imbalance. ${ }^{25}$

\section{FIXED-EFFECTS META-ANALYSIS}

We aggregated the association summary statistics from the eight data sets using a fixed-effects inverse-variance metaanalysis in METAL. ${ }^{26}$ We applied a genomic control correction to each association summary statistic. Heterogeneity
A

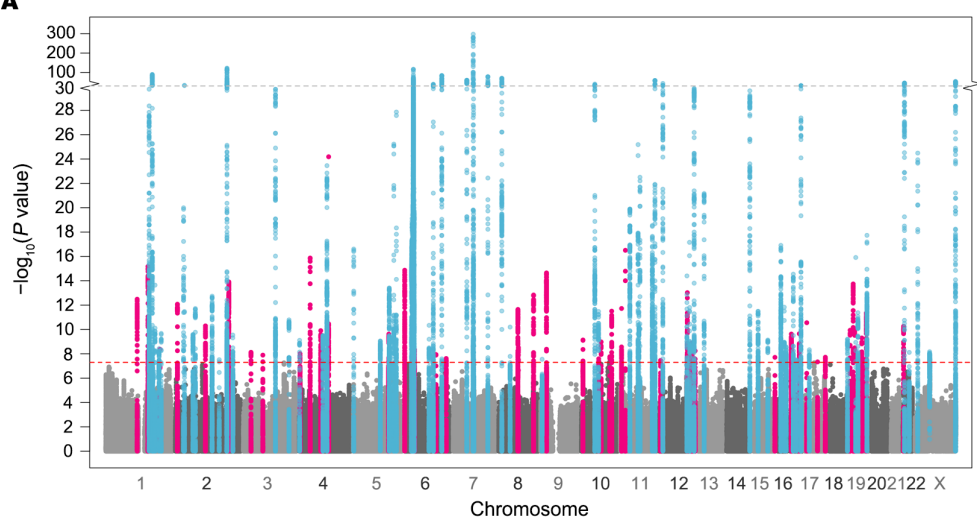

B

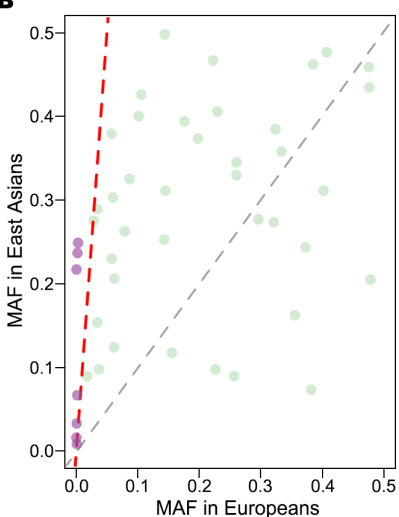

Figure 1 Summary of meta-analysis association results and comparison of MAFs for lead variants within the 46 novel loci between East Asians and Europeans. (A) Manhattan plot of genome-wide association meta-analysis results from 208370 SLE East Asians including 13377 SLE cases and 194993 controls. Minus log10-transformed association p values ( $y$-axis) are plotted along chromosomal positions (x-axis). Known and novel loci are highlighted in light blue and pink, respectively. The red dashed line denotes the genome-wide association significance threshold of $p=5 \times 10^{-8}$. The grey dashed line represents $p=10^{-30}$, at which the $y$-axis breaks. (B) Comparison of MAFs of lead variants within the 46 novel loci between East Asians (y-axis) and non-Finnish Europeans (x-axis) in the Genome Aggregation Database (gnomAD) v3. Variants with more than 10 times higher MAFs in East Asians are coloured purple above a red dashed line. MAF, minor allele frequency. 
Systemic lupus erythematosus

Table 1 Association results for the 46 novel susceptibility loci for systemic lupus erythematosus

\begin{tabular}{|c|c|c|c|c|c|c|c|c|c|c|c|c|c|}
\hline Region & CHR & Position & Variant & EA & NEA & EAF & OR & SE & $P$ value & $\mathrm{I}^{2}$ & $\mathbf{P}_{\text {Het }}$ & $\mathrm{N}$ & Nearest gene \\
\hline 1 & 1 & 117043302 & rs9651076 & A & G & 0.431 & 1.117 & 0.015 & $3.26 \mathrm{E}-13$ & 10.7 & 0.347 & 208370 & CD58 \\
\hline 2 & 1 & 157108159 & rs116785379 & C & G & 0.107 & 1.211 & 0.024 & $6.68 \mathrm{E}-16$ & 43.7 & 0.114 & 208370 & ETV3 \\
\hline 7 & 1 & 201979455 & rs3806357 & A & G & 0.251 & 1.106 & 0.017 & $4.25 \mathrm{E}-09$ & 0.0 & 0.672 & 208370 & ELF3 \\
\hline 9 & 2 & 7573079 & rs75362385 & $\mathrm{T}$ & G & 0.321 & 0.887 & 0.017 & $8.40 \mathrm{E}-13$ & 68.3 & 0.007 & 208370 & LOC100506274 \\
\hline 14 & 2 & 111877174 & rs73954925 & C & G & 0.878 & 1.169 & 0.024 & $5.11 \mathrm{E}-11$ & 56.4 & 0.043 & 208370 & $B C L 2 L 11$ \\
\hline 18 & 2 & 198929806 & rs7572733 & $\mathrm{T}$ & $\mathrm{C}$ & 0.260 & 1.143 & 0.017 & $1.25 \mathrm{E}-14$ & 0.0 & 0.647 & 208370 & PLCL1 \\
\hline 20 & 3 & 28072086 & rs438613 & $\mathrm{T}$ & $\mathrm{C}$ & 0.588 & 0.920 & 0.014 & $7.52 \mathrm{E}-09$ & 69.4 & 0.006 & 208370 & LINC01980 \\
\hline 21 & 3 & 72225916 & rs7637844 & A & $\mathrm{C}$ & 0.871 & 0.877 & 0.023 & $1.28 \mathrm{E}-08$ & 0.0 & 0.906 & 208370 & LINC00870 \\
\hline 25 & 4 & 2700844 & rs231694 & $\mathrm{T}$ & C & 0.380 & 1.111 & 0.018 & $9.71 \mathrm{E}-09$ & 23.7 & 0.269 & 57253 & FAM193A \\
\hline 26 & 4 & 40307587 & rs113284964 & G & GCTTC & 0.371 & 1.134 & 0.015 & $1.35 \mathrm{E}-16$ & 67.2 & 0.009 & 208370 & LINC02265 \\
\hline 27 & 4 & 79644279 & rs6533951 & A & G & 0.350 & 1.111 & 0.016 & $1.25 \mathrm{E}-10$ & 61.4 & 0.024 & 208370 & LINC01094 \\
\hline 28 & 4 & 84146996 & rs6841907 & $\mathrm{T}$ & $\mathrm{C}$ & 0.729 & 0.906 & 0.016 & $1.10 \mathrm{E}-09$ & 43.5 & 0.115 & 208370 & COQ2 \\
\hline 31 & 4 & 109061618 & rs58107865 & C & G & 0.227 & 0.802 & 0.021 & $6.57 \mathrm{E}-25$ & 1.1 & 0.409 & 208370 & LEF1 \\
\hline 34 & 5 & 131120338 & rs370449198 & A & $A C$ & 0.922 & 0.721 & 0.060 & $4.41 \mathrm{E}-08$ & 0.0 & 0.408 & 187562 & FNIP1 \\
\hline 35 & 5 & 131829578 & rs 2549002 & A & C & 0.682 & 0.905 & 0.016 & $2.40 \mathrm{E}-10$ & 20.6 & 0.279 & 208370 & IRF1 \\
\hline 40 & 6 & 243302 & rs9503037 & A & G & 0.693 & 0.881 & 0.016 & $1.36 \mathrm{E}-15$ & 42.3 & 0.123 & 208370 & LOC285766 \\
\hline 43 & 6 & 36715031 & rs34868004 & CA & C & 0.225 & 1.104 & 0.017 & $4.46 \mathrm{E}-09$ & 40.7 & 0.134 & 208370 & CPNE5 \\
\hline 46 & 6 & 116690849 & rs9488914 & $\mathrm{T}$ & C & 0.920 & 0.862 & 0.026 & $1.14 \mathrm{E}-08$ & 65.3 & 0.013 & 208370 & DSE \\
\hline 48 & 6 & 154570651 & rs9322454 & A & G & 0.659 & 1.090 & 0.015 & $2.42 \mathrm{E}-08$ & 0.0 & 0.430 & 208370 & IPCEF1 \\
\hline 54 & 8 & 71330166 & rs142937720 & A & AAGTGGCC & 0.383 & 0.894 & 0.016 & $2.27 \mathrm{E}-12$ & 67.9 & 0.008 & 208370 & NCOA2 \\
\hline 55 & 8 & 72894959 & rs17374162 & A & G & 0.411 & 0.917 & 0.015 & $3.02 \mathrm{E}-09$ & 35.7 & 0.169 & 208370 & MSC-AS1 \\
\hline 56 & 8 & 129425593 & rs16902895 & A & G & 0.678 & 1.122 & 0.016 & $1.48 \mathrm{E}-13$ & 0.0 & 0.801 & 208370 & LINC00824 \\
\hline 58 & 9 & 21267087 & rs7858766 & $\mathrm{T}$ & C & 0.538 & 1.139 & 0.016 & $2.25 \mathrm{E}-15$ & 0.0 & 0.825 & 208370 & IFNA22P \\
\hline 59 & 10 & 5910746 & rs77448389 & A & G & 0.913 & 0.855 & 0.025 & $7.30 \mathrm{E}-10$ & 0.0 & 0.584 & 208370 & ANKRD16 \\
\hline 62 & 10 & 64411288 & rs10995261 & $\mathrm{T}$ & $C$ & 0.240 & 0.909 & 0.017 & $2.57 \mathrm{E}-08$ & 43.9 & 0.113 & 208370 & ZNF365 \\
\hline 63 & 10 & 73466709 & rs10823829 & $\mathrm{T}$ & C & 0.718 & 0.910 & 0.016 & $1.05 \mathrm{E}-09$ & 0.0 & 0.771 & 208370 & $\mathrm{CDH} 23$ \\
\hline 64 & 10 & 105677911 & rs111447985 & A & C & 0.073 & 1.172 & 0.028 & $1.72 \mathrm{E}-08$ & 0.0 & 0.526 & 208370 & STN1 \\
\hline 65 & 10 & 112664114 & rs58164562 & $\mathrm{T}$ & $\mathrm{C}$ & 0.748 & 0.892 & 0.016 & $3.14 \mathrm{E}-12$ & 33.3 & 0.186 & 208370 & $B B I P 1$ \\
\hline 66 & 11 & 4113200 & rs3750996 & A & G & 0.834 & 1.167 & 0.022 & $1.89 \mathrm{E}-12$ & 0.0 & 0.522 & 208370 & STIM1 \\
\hline 67 & 11 & 18362382 & rs77885959 & $\mathrm{T}$ & G & 0.978 & 1.694 & 0.062 & $3.16 \mathrm{E}-17$ & 0.0 & 0.511 & 204433 & GTF2H1 \\
\hline 74 & 12 & 4140876 & rs2540119 & $\mathrm{T}$ & C & 0.544 & 1.086 & 0.015 & $3.51 \mathrm{E}-08$ & 44.9 & 0.106 & 208370 & PARP11 \\
\hline 77 & 12 & 103916080 & rs6539078 & $\mathrm{T}$ & $\mathrm{C}$ & 0.591 & 0.894 & 0.015 & $9.49 \mathrm{E}-14$ & 0.0 & 0.916 & 208370 & LOC105369945 \\
\hline 79 & 12 & 121368518 & rs3999421 & A & $\mathrm{T}$ & 0.506 & 0.910 & 0.016 & $1.29 \mathrm{E}-09$ & 47.3 & 0.091 & 208370 & XLOC_009911 \\
\hline 81 & 12 & 133040182 & rs200521476 & G & GCATCAC & 0.812 & 0.875 & 0.023 & $5.66 \mathrm{E}-09$ & 26.7 & 0.235 & 208370 & FBRSL1 \\
\hline 86 & 15 & 101529012 & rs35985016 & A & G & 0.930 & 0.843 & 0.030 & $1.95 \mathrm{E}-08$ & 0.0 & 0.897 & 204433 & LRRK1 \\
\hline 90 & 16 & 50089207 & rs11288784 & G & GT & 0.365 & 0.902 & 0.016 & $2.38 \mathrm{E}-10$ & 0.0 & 0.664 & 208370 & HEATR3 \\
\hline 93 & 16 & 79745672 & rs11376510 & G & GT & 0.737 & 0.898 & 0.017 & $2.23 E-10$ & 0.0 & 0.719 & 208370 & MAFTRR \\
\hline 95 & 17 & 7240391 & rs61759532 & $\mathrm{T}$ & $\mathrm{C}$ & 0.076 & 1.235 & 0.032 & $2.79 \mathrm{E}-11$ & 24.9 & 0.247 & 208370 & ACAP1 \\
\hline 97 & 17 & 47468020 & rs2671655 & $\mathrm{T}$ & $\mathrm{C}$ & 0.651 & 1.087 & 0.015 & $4.60 \mathrm{E}-08$ & 0.0 & 0.756 & 208370 & LOC10272459 \\
\hline 98 & 17 & 76373179 & rs113417153 & $\mathrm{T}$ & C & 0.193 & 0.893 & 0.020 & $1.90 \mathrm{E}-08$ & 2.1 & 0.403 & 208370 & PGS1 \\
\hline 100 & 18 & 77386912 & rs118075465 & A & G & 0.147 & 1.140 & 0.020 & $1.16 \mathrm{E}-10$ & 0.0 & 0.543 & 208370 & LOC284241 \\
\hline 101 & 19 & 948532 & rs2238577 & $\mathrm{T}$ & $\mathrm{C}$ & 0.455 & 0.885 & 0.016 & $1.83 \mathrm{E}-14$ & 60.8 & 0.026 & 208370 & ARID3A \\
\hline 102 & 19 & 6697088 & rs5826945 & A & $\mathrm{T}$ & 0.929 & 0.836 & 0.028 & $9.67 \mathrm{E}-11$ & 50.0 & 0.075 & 208370 & C3 \\
\hline 105 & 19 & 33072768 & rs12461589 & $\mathrm{T}$ & C & 0.248 & 0.898 & 0.017 & $5.00 \mathrm{E}-10$ & 0.0 & 0.510 & 208370 & PDCD5 \\
\hline 106 & 19 & 49851746 & rs33974425 & CCAGCTGCAT & C & 0.702 & 1.120 & 0.016 & $4.40 \mathrm{E}-12$ & 42.6 & 0.121 & 208370 & TEAD2 \\
\hline 108 & 22 & 18649356 & rs4819670 & $\mathrm{T}$ & $\mathrm{C}$ & 0.210 & 1.151 & 0.022 & $5.53 \mathrm{E}-11$ & 0.0 & 0.650 & 208370 & USP18 \\
\hline
\end{tabular}

CHR, chromosome; EA, effect allele; EAF, effect allele frequency; $I^{2}$, genetic heterogeneity $I^{2}$ statistics at scale of $0 \%$ to $100 \%$; $N$, study sample size; NEA, non-effect allele; OR, Odds ratio; $\mathrm{P}_{\text {Het' }}$

P-values for the $\chi^{2}$ test of genetic heterogeneity; Region, unique ID for genomic region; ;SE, Standard error of odds ratio.

in allelic effect sizes among data sets was assessed using Cochran's Q statistic. We excluded genetic variants available in only a single data set. We defined SLE susceptibility loci by merging \pm 250 kilobases $(\mathrm{kb})$ windows around genomewide associated variants to ensure that lead single nucleotide polymorphisms (SNPs) were at least $500 \mathrm{~kb}$ apart. We defined lead variants as the most significant SLE-associated variant within each locus. A locus was considered novel if the lead SNP was at least $500 \mathrm{~kb}$ away from any previously reported SLE-associated variants.

\section{Approximate conditional association analysis}

To dissect distinct association signals at each SLE locus, we performed an approximate conditional analysis using GCTA $\mathrm{COJO}^{27}$ with genome-wide meta-analysis summary statistics based on linkage disequilibrium (LD) estimated from 7021 unrelated Chinese controls. The Chinese reference individuals for LD calculation were retrieved from the Chinese study using the Illumina Infinium Global Screening Array data (online supplemental table 1), excluding first-degree and second-degree relatives. 

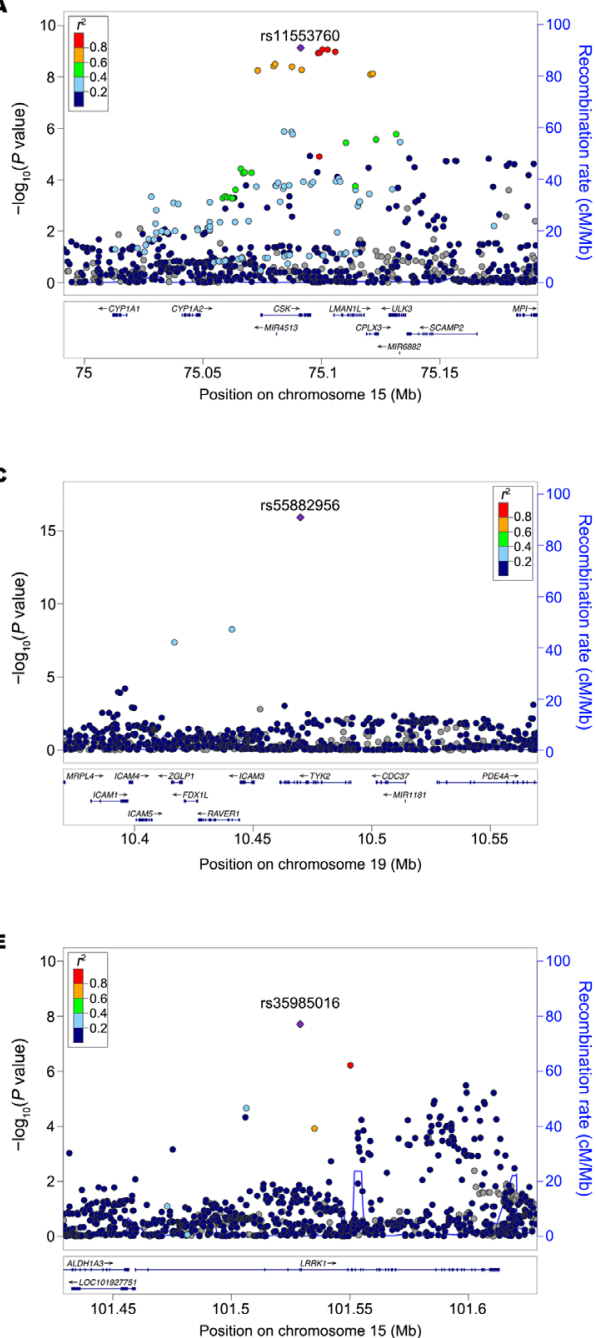
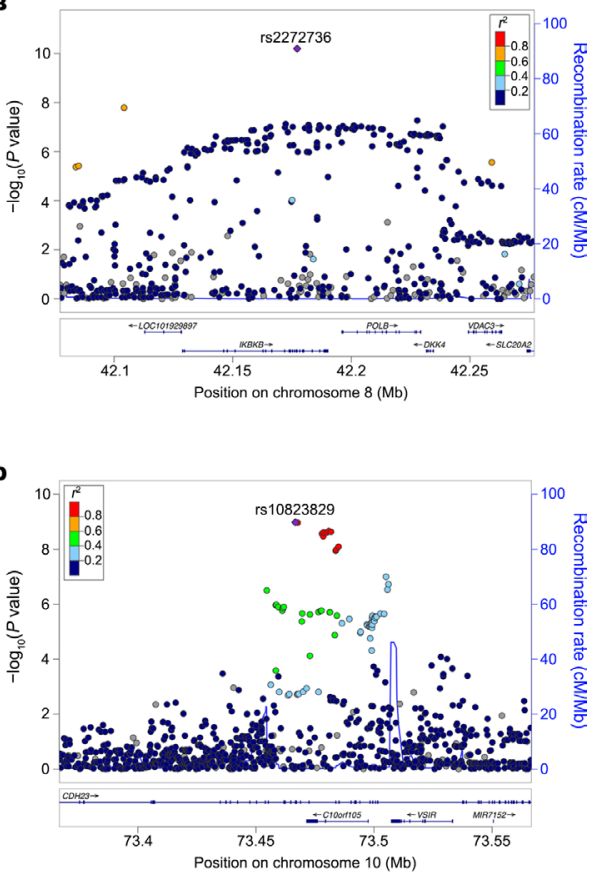

Figure 2 New lead exonic variants identified at three known (CSK, IKBKB and TYK2) and two novel (CHD23 and LRRK1) loci. (A) rs11553760 (synonymous variant) at CSK. (B) rs2272736 (p.Arg303Gln, missense variant) at IKBKB. (C) rs55882956 (p.Arg703Trp, missense variant) at TYK2. (D) rs10823829 (synonymous variant) at CHD23. (E) rs35985016 (p.Lys203Glu, missense variant) at LRRK1. The lead SNP is labelled as purple diamond. The LD is estimated from 7021 Chinese samples. LD, linkage disequilibrium; Mb, megabases; SNP, single nucleotide polymorphism.

\section{Bayesian statistical fine-mapping analysis}

To prioritise causal variants in SLE susceptibility loci, a statistical fine-mapping analysis was performed using FINEMAP v1.4 software, ${ }^{28}$ with meta-analysis z-scores and LD matrices estimated from the 7021 Chinese reference individuals. We used default priors and parameters in FINEMAP, assuming at most five causal signals in the $\pm 250 \mathrm{~kb}$ region around a lead variant at each SLE locus. FINEMAP computed a posterior probability (PP) for each genetic variant being the true putative causal variant. For each association signal, we ranked the candidate putative causal variants in a descending order of their PPs, and then built a 95\% credible set of causal variants by including the ordered variants until their cumulative PP reached 0.95 .

\section{Heritability estimation by LD score regression}

Overall SLE heritability $h^{2}$ explained by genome-wide variants was estimated using the LD score regression model ${ }^{29}$ with LD scores ${ }^{18}$ from the 1KGP East Asian descendants, based on an SLE population prevalence of $0.03 \%$ in East Asian populations. ${ }^{1}$ SLE heritability estimate was further partitioned according to known and novel SLE loci using stratified LD score regression. ${ }^{30}$ The boundary of each SLE locus was arbitrarily defined as $\pm 500 \mathrm{~kb}$ flanking the lead SLE-risk variant.

\section{Genetic correlation between SLE and other traits by LD score} regression

We calculated genetic correlations between 98 traits (39 diseases $^{17}$ and 59 quantitative traits ${ }^{31}$ and SLE by using bivariate LD score regression. ${ }^{32}$ We used the LD scores ${ }^{18}$ from the 1KGP East Asian descendants, limited the genetic variants to the HapMap3 SNPs and removed the variants with extended human leucocyte antigen (HLA) region (chromosome 6: 25 to 34 megabases $(\mathrm{Mb}))$.

\section{Patient and public involvement}

Patients and the public were not involved in the design or analysis of this study.

\section{RESULTS}

\section{Identification of $\mathbf{4 6}$ novel SLE susceptibility loci}

We performed a large genome-wide association meta-analysis in 13377 SLE cases and 194993 controls of East Asians (online supplemental table 1). To the best of our knowledge, this is the largest genetic association study of SLE to date. The effective sample size $\left(\mathrm{N}_{\mathrm{eff}}=50072\right)$ is three-fold and four-fold larger than 
A

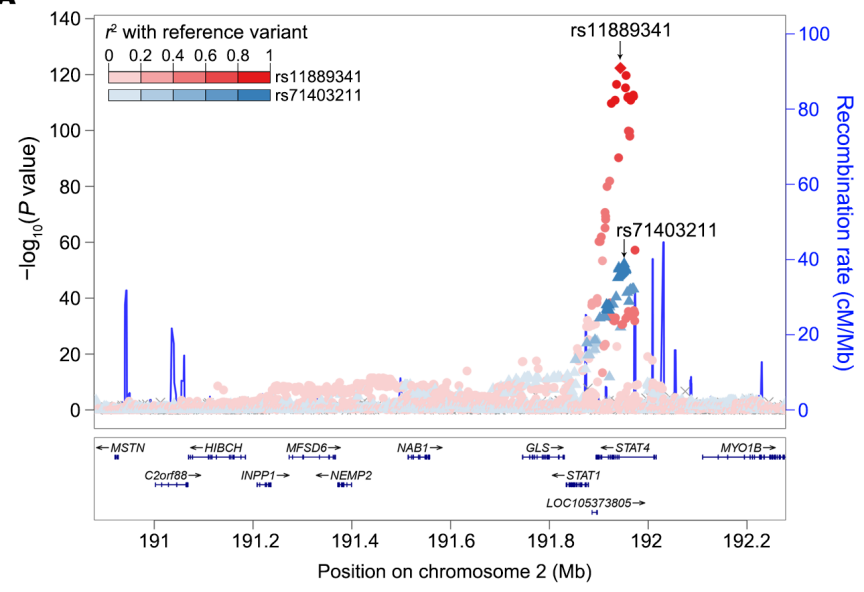

B

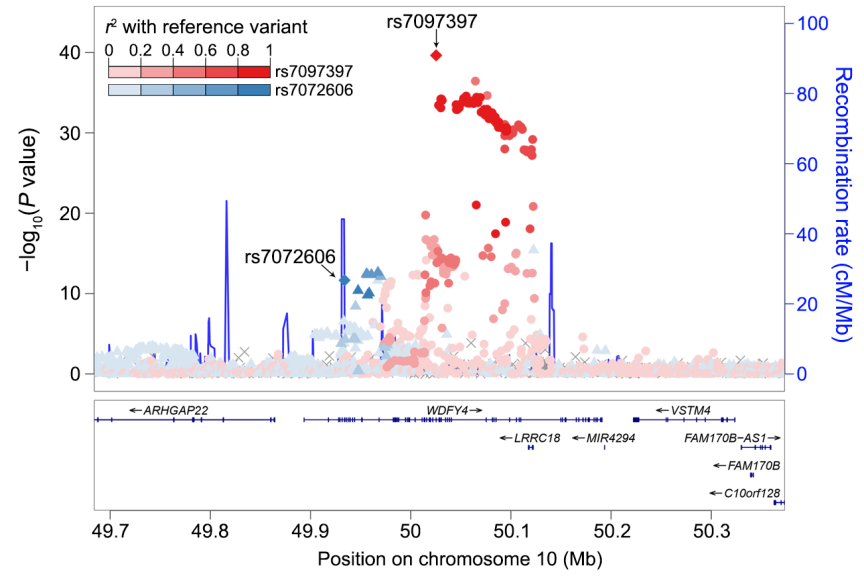

C

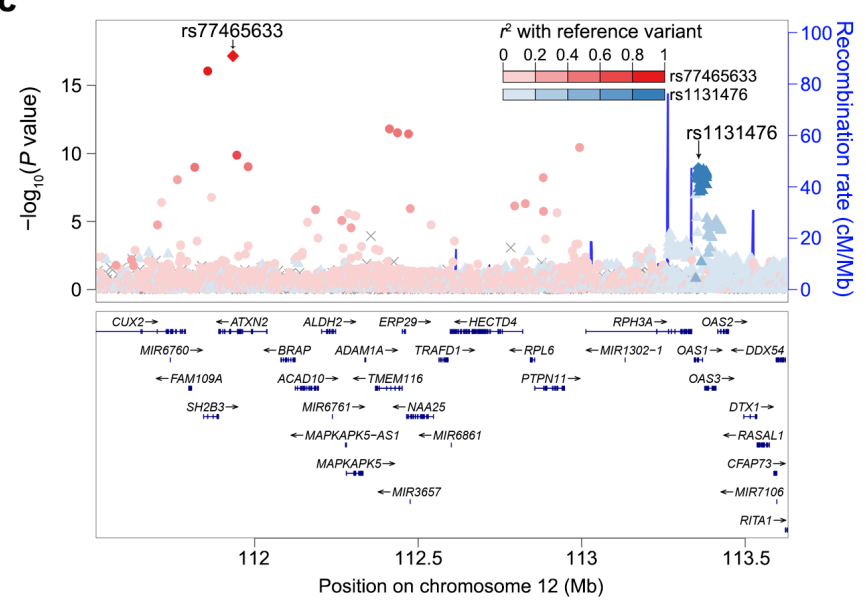

Figure 3 Two independent association signals identified. (A) At two intronic variants within known STAT4 locus. (B) At known (rs7097397, p.Arg1816Gln) and new (rs7072606, p.Ser214Pro) missense variants within WDFY4 locus. (C) A known intronic variant within ATXN2 gene and a new (rs1131476, p.Ala352Thr) missense variant within OAS1 gene. The lead and secondary index variants are labelled in diamond. The lead variant and its LD proxies are in red while the secondary signal index variant and its LD proxies are in blue. The LD is estimated from 7021 Chinese samples. LD, linkage disequilibrium; Mb, megabases.

that of the largest published trans-ancestry ${ }^{9}$ and East Asian ${ }^{11}$ meta-analyses, respectively.

We tested associations for 11270530 genetic variants in a fixed-effects meta-analysis. A quantile-quantile plot showed that test statistics were well-calibrated, with a genomic-control inflation factor $\lambda_{\mathrm{GC}}=1.06$ (indicating that ancestry effects had been well controlled; online supplemental figure 1). LD score regression ${ }^{29}$ showed that polygenic effects $(89.4 \%)$, rather than biasses, primarily caused the inflation residual (estimated mean $\chi^{2}=1.32$ and LD-score intercept $=1.03$ ).

We detected 26379 genetic variants associated with SLE at $\mathrm{p}<5 \times 10^{-8}$ within 113 loci (figure $1 \mathrm{~A}$ and online supplemental table 2), of which 46 were novel (table 1). The pairwise LD between lead variants was low $\left(\mathrm{LD}^{2}<0.002\right)$. For seven novel loci, MAFs of the lead SNPs were 10-fold higher in East Asians than in Europeans (figure 1B). Two of them and their LD neighbours $\left(r^{2} \geq 0.2\right.$ in either East Asians or Europeans) would be undetectable in Europeans with the same effective sample size and risk magnitude due to low statistical power $(<10 \%$; online supplemental table 3).

\section{Associations at exonic variants}

The meta-analysis identified lead missense variants in two novel loci (CHD23 and LRRK1; figure 2A,B and online supplemental table 2). In addition, we detected three new exonic variants (including two missense variants) within the reported SLE loci including CSK (rs11553760), IKBKB (rs2272736) and TYK2 (rs55882956) genes (figure 2C-E and online supplemental table 2 ). They were not correlated with previously reported exonic variants within the same genes $\left(\mathrm{LD} \mathrm{r}^{2}<0.02\right.$ in East Asians or Europeans; online supplemental table 4), suggesting possible allelic heterogeneity of these genes. We replicated four known associations for missense variants at AHNAK2 (rs2819426), ${ }^{33}$ IRAK1 (rs1059702), ${ }^{34}$ NCF2 (rs13306575) and WDFY4 (rs7097397; online supplemental table 2). ${ }^{3536}$

\section{Secondary association signals within SLE loci}

To dissect the source of association signals at each locus, we conducted an approximate conditional analysis using GCTA ${ }^{27}$ with meta-analysis summary statistics and LD estimates from 7021 unrelated Chinese controls. We acknowledge the limitations of using LD estimation from a single population for a meta-analysis of diverse East Asians. We identified a total of 233 independent association signals with conditional $\mathrm{p}<5 \times 10^{-8}, 169$ of which arose from non-HLA regions (online supplemental table 5 ). We observed from two to four signals at each of 28 non-HLA loci (including seven novel loci). For example, we discovered two distinct association signals within the known STAT4 locus, including the previously reported SNP rs11889341 ${ }^{12}$ and the new insert-deletion variant (indel) rs71403211 (figure 3A). For the 46 novel loci, we discovered 55 distinct signals (online supplemental table 5 and figure 2). We noticed that most of the signal index variants $(n=190$, $82 \%$ ) are common (MAF $\geq 5 \%$ ) with modest effects (online supplemental table 5).

Approximate conditional analysis detected two novel missense variants at WDFY4 and OAS1 genes. We detected two distinct signals within WDFY4, including the known (rs7097397) ${ }^{37}$ and a new (rs7072606) missense variant (LD r ${ }^{2}=0.02$ between two variants in East Asians), which suggests allelic heterogeneity at this locus (figure $3 \mathrm{~B}$ ). We provided for the first time genomewide association evidence at a missense variant within OAS1 (rs1131476, LD $\mathrm{r}^{2}=0.78$ with rs1051042, which is a known missense variant but only exhibited suggestive significance with SLE in previous study, ${ }^{33}$ figure $3 \mathrm{C}$ and online supplemental table $5)$. 

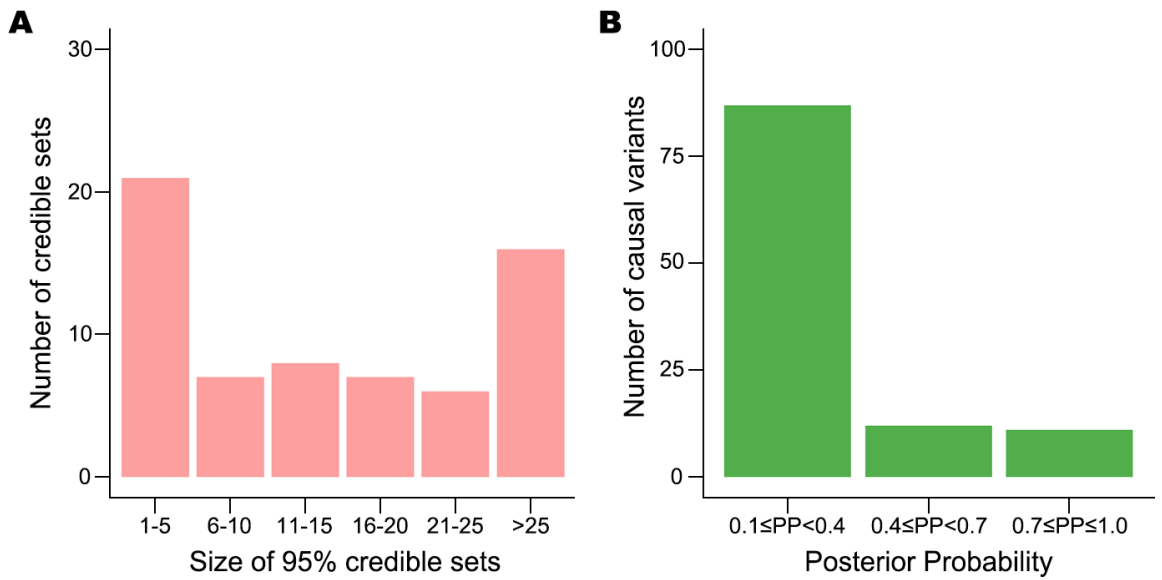

Figure 4 Results of statistical fine-mapping analysis. (A) Number of $95 \%$ credible sets of putative causal variants, binned by their sizes. (B) Number of potential causal variants with posterior probabilities $(P P) \geq 0.1$, which are considered to be the true causal variants.

\section{Prioritisation of causal variants}

To prioritise putative causal variants, we conducted a Bayesian statistical fine-mapping analysis for 111 loci using FINEMAP ${ }^{28}$ after excluding complex associations involving the HLA and 7q11.23. We found exactly the same number of association signals in 57 loci between FINEMAP causal configuration with the highest posterior probability and the GCTA approximate conditional test. To be conservative, we only summarised the statistical fine-mapping results for these 57 regions, which contained 65 association signals (online supplemental table 6).

For each signal, we built a credible set of putative causal variants with a $95 \%$ probability of including the true causal variants. The size of 28 credible sets was small (size $\leq 10$; figure $4 \mathrm{~A}$ ). Among the 110 putative causal variants with posterior probability $\geq 0.1$ (figure $4 \mathrm{~B}$ ), we found four coding variants (3.6\%), which implies that most of these associations are probably induced by non-coding causal variants. The prioritised variants are available to be tested as potential targets in perturbation experiments. For example, the allele-specific regulatory activity of the intronic variant (rs10036748) with the highest posterior probability $(0.387)$ in the TNIP1 locus was recently experimentally characterised in SLE. ${ }^{38}$

We pinpointed a single most likely causal variant with high confidence (posterior probability $\geq 0.8$ ) for four known (ATXN2, BACH2, DRAM1/WASHC3 and NCF2) and six novel (17p13.1, ELF3, GTF2H1, LRRK1, LOC102724596/PHB and STIM1) loci (online supplemental table 6). For example, we prioritised rs61759532 as a putative causal variant at the novel $17 \mathrm{p} 13.1$ locus $(\mathrm{PP}=0.999)$. This variant is located in an intron of ACAP1, which encodes a key regulator of integrin traffic for cell adhesion and migration. ${ }^{39}$

\section{SNP-BASED HERITABILITY}

To assess the proportion of phenotypic variance explained by common variants, we applied LD score regression ${ }^{29}$ to the metaanalysis results. Assuming a population prevalence of $0.03 \%$ for SLE, ${ }^{1}$ we estimated the liability-scale SNP-based heritability from all non- $H L A$ variants as $\mathrm{h}_{\mathrm{SNP}}^{2}=7.24 \%$ ( $\left.\mathrm{SE}=0.78 \%\right)$. The 66 known and 46 novel non-HLA loci explained $62.6 \%$ (SE $=4.9 \%)$ and $22.1 \%(\mathrm{SE}=2.6 \%)$ of this overall SNP-based heritability, respectively.

\section{Genetic correlation with other diseases/traits}

To explore shared genetics between SLE and various traits, we calculated genetic correlations of SLE with 39 complex diseases and 59 quantitative traits in Biobank Japan participants using bivariate LD score regression ${ }^{32}$ (online supplemental table 7). As expected, we detected significant positive genetic correlations between SLE and two other autoimmune diseases: rheumatoid arthritis $\left(\mathrm{r}_{\mathrm{g}}=0.437\right)$ and Graves' disease $\left(\mathrm{r}_{\mathrm{g}}=0.318\right)$. In addition, we found unreported genetic correlations $(\mathrm{FDR}<0.05)$ with albumin/globulin ratio $\left(r_{\mathrm{g}}=-0.242\right)$ and non-albumin protein $\left(\mathrm{r}_{\mathrm{g}}=0.238\right)$.

\section{DISCUSSION}

Here, we carried out the largest-ever genome-wide association meta-analysis for SLE and identified 113 risk loci including 46 novel regions for SLE in 208370 East Asians including 13377 SLE cases and 194993 controls. This study revealed new genetic predispositions for SLE and generated hypotheses for further studies to investigate diseases functional mechanisms.

Epidemiological studies have found the higher prevalence of SLE in East Asians and heterogeneous disease manifestations across ethnicities. ${ }^{15} 16$ Previous investigations suggested genetics might explain the phenotypic heterogeneity. ${ }^{9}$ We observed that the MAFs of the index variants for several novel genetic associations were much higher in East Asians than in Europeans. Specifically, we suggested two novel loci were more likely specific to East Asians. These findings might help explain the genetic basis of SLE phenotypic heterogeneity between East Asians and Europeans. The results reinforce the power of large-scale genetic association for genetic discovery of SLE in relatively less studied populations.

We identified 11 exonic variants including two missense variants within novel loci CHD23 and LRRK1, four novel missense variants within known SLE loci IKBKB, ${ }^{9}$ TYK $2,{ }^{9}$ WDFY ${ }^{37}$ and OAS1, ${ }^{33}$ and three known missense variants within known AHNAK2, ${ }^{33}$ IRAK1 $1^{34}$ and NCF2. ${ }^{35} 36$ These findings suggested allelic heterogeneity within several of these loci and highlighted the disease-risk effects of genes AHNAK2, CSK, IKBKB, IRAK1, NCF2, OAS1, TYK2 and WDFY4 within eight known loci, and CDH23 and LRRK1 within two novel loci which potentially alter gene product activity in an allele-specific manner. The novel gene CHD23 plays a role in cell migration ${ }^{40}$ while LRRK1 encodes a multiple-domain leucine-rich repeat kinase. A previous study observed that LRRK1-deficient mice exhibited a profound defect in B-cell proliferation and survival and impaired B-cell receptor-mediated NF- $\mathrm{KB}$ activation, ${ }^{41}$ which suggested that the association within this region might confer the risk of SLE through modulating the NF- $\mathrm{kB}$ pathway and the activities of $\mathrm{B}$ cells. We noted that the Bayesian statistical fine-mapping analysis prioritised the lead missense variant rs35985016 as the most likely putative 
causal variant for this association. This variant is highly frequent in our study individuals but is rare in Europeans. The molecular mechanisms in SLE risk worthy further investigations.

In the present study, we localised the putative causal variants for SLE genetic association in high resolution. Our findings indicated that the putative causal variants for the majority of SLE associations were non-coding variants. We provided targets of candidate putative causal variants with high confidence for several SLE loci. These findings are worthy for further exploration in functional experiments. We showed the regulatory effect of one of the putative causal variants in an accompanied paper. We acknowledged the limitation of a small LD reference panel from single population in the Bayesian statistical fine-mapping analysis.

We found for the first time the significant genetic correlations between SLE, albumin/globulin ratio and non-albumin protein. These findings might reflect the renal complications commonly developed in SLE patients who have been reported to have significantly lower albumin/globulin ratio and higher serum globulin than healthy controls in epidemiological studies. ${ }^{42}$ These shared genetic basis findings might suggest a common pathway underlying the SLE risk and kidney function in addition to the direct damage of SLE autoantibodies on kidney.

In summary, we detected 46 novel loci for SLE risk in the largest meta-analysis and prioritised putative causal variants for 65 causal signals. This study highlights the power of large-scale genetic association study in East Asian populations. The findings reveal the genetic predispositions for SLE and provide clues for further the investigation of disease mechanisms.

\section{Author affiliations}

'Department of Dermatology, First Affiliated Hospital, Anhui Medical University, Hefei, Anhui, China

Institute of Dermatology, Anhui Medical University, Hefei, Anhui, China

${ }^{3}$ Key Lab of Dermatology, Ministry of Education (Anhui Medical University), Hefei, Anhui, China

${ }^{4}$ Inflammation and Immune Mediated Diseases Laboratory of Anhui Province, Hefei, Anhui, China

5Department of Dermatology, China-Japan Friendship Hospital, Beijing, China ${ }^{6}$ Department of Biostatistics, Center for Statistical Genetics, University of Michigan, Ann Arbor, Michigan, USA

${ }^{7}$ Department of Biology and Department of Life and Nanopharmaceutical Sciences, Kyung Hee University, Seoul, Korea

${ }^{8}$ Laboratory for Bone and Joint Diseases, RIKEN Center for Medical Sciences, Kanagawa, Japan

${ }^{9}$ Laboratory for Statistical and Translational Genetics Analysis, RIKEN Center for Integrative Medical Sciences, Kanagawa, Japan

${ }^{10}$ Department of Orthopaedic Surgery, Graduate School of Medical Sciences, Kyushu University, Fukuoka, Japan

${ }^{11}$ Department of Rheumatology, Hanyang University Hospital for Rheumatic Diseases, Seoul, Korea

${ }^{12}$ Hanyang University Institute for Rheumatology Research, Seoul, Korea

${ }^{13}$ Division of Molecular Pathology, Department of Cancer Biology, Institute of

Medical Science, The University of Tokyo, Tokyo, Japan

${ }^{14}$ Koga Hospital 21, Fukuoka, Japan

${ }^{15}$ Department of Epidemiology and Biostatistics, School of Public Health, Anhui Medical University, Hefei, Anhui, China

${ }^{16}$ Department of Orthopedic Surgery, Keio University School of Medicine, Tokyo, Japan

${ }^{17}$ Center for Autoimmune Genomics and Etiology (CAGE), Cincinnati Children's Hospital Medical Center, Cincinnati, Ohio, USA

${ }^{18}$ Department of Orthopaedic Medical Engineering, Osaka University Graduate School of Medicine, Osaka, Japan

${ }^{19}$ Division of Genome Research, Center for Genome Science, National Institute of Health, Osong Health Technology Administration Complex, Cheongju, Korea ${ }^{20}$ Department of Rheumatology \& Clinical Immunology, Sapporo City General Hospital, Hokkaido, Japan

${ }^{21}$ Divisions of Genetics and Rheumatology, Department of Medicine, Brigham and Women's Hospital, Harvard Medical School, Boston, Massachusetts, USA

${ }^{22}$ Center for Data Sciences, Harvard Medical School, Boston, Massachusetts, USA

${ }^{23}$ Program in Medical and Population Genetics, Broad Institute of MIT and Harvard, Cambridge, Massachusetts, USA
${ }^{24}$ Department of Internal Medicine, Dong-A University Hospital, Busan, Korea

${ }^{25}$ Department of Orthopaedic Surgery, Faculty of Medicine and Graduate School of

Medicine, Hokkaido University, Hokkaido, Japan

${ }^{26}$ Division of Rheumatology, Department of Internal Medicine, Chonnam National

University Medical School and Hospital, Gwangju, Korea

${ }^{27}$ Department of Rheumatology, Endocrinology and Nephrology, Faculty of Medicine and Graduate School of Medicine, Hokkaido University, Hokkaido, Japan

${ }^{28}$ Division of Rheumatology, Department of Internal Medicine, Chungnam National

University Hospital, Daejeon, Korea

${ }^{29}$ Department of Internal Medicine and Rheumatology, National Hospital

Organization, Kyushu Medical Center, Fukuoka, Japan

${ }^{30}$ Division of Rheumatology, Department of Internal medicine, Kyungpook National

University Hospital, Daegu, Korea

${ }^{31}$ Department of Orthopaedic Surgery, Graduate School of Medicine, Chiba University, Chiba, Japan

${ }^{32}$ Department of Rheumatology, Ajou University School of Medicine, Suwon, Korea

${ }^{33}$ Shanghai Institute of Rheumatology, Renji Hospital, Shanghai Jiao Tong University,

School of Medicine (SJTUSM), Shanghai, China

${ }^{34}$ Department of Internal Medicine, Yonsei University College of Medicine, Seoul,

Korea

${ }^{35}$ Niigata University Health Administration Center, Niigata, Japan

${ }^{36}$ Department of Rheumatology, Catholic University of Daegu School of Medicine,

Daegu, Korea

${ }^{37}$ Department of Medical Education, Kyushu University Graduate School of Medical

Sciences, Fukuoka City, Japan

${ }^{38}$ Department of Dermatology, Peking University Shenzhen Hospital, Shenzhen,

Guangdong, China

${ }^{39}$ Shenzhen Key Laboratory for Translational Medicine of Dermatology, Shenzhen

Peking University - The Hong Kong University of Science and Technology Medical

Center, Shenzhen, Guangdong, China

${ }^{40}$ Department of Orthopaedic Surgery, Faculty of Life Sciences, Kumamoto University, Kumamoto, Japan

${ }^{41}$ Division of Rheumatology, Department of Internal Medicine, Keio University School of Medicine, Tokyo, Japan

${ }^{42}$ Institute of Rheumatology, Tokyo Women's Medical University, Tokyo, Japan

${ }^{43}$ Department of Paediatrics and Adolescent Medicine, The University of Hong Kong,

Pok Fu Lam, Hong Kong, China

${ }^{44}$ Department of Rheumatology \& Clinical Immunology, Saitama Medical Center, Saitama Medical University, Saitama, Japan

${ }^{45}$ Department of Rheumatology, Faculty of Medicine, Saga University, Saga, Japan

${ }^{46}$ Department of Internal Medicine and Rheumatology, Juntendo University School of

Medicine, Tokyo, Japan

${ }^{47}$ Hokkaido Medical Center for Rheumatic Disease, Hokkaido, Japan

${ }^{48}$ Department of Orthopaedic Surgery, Showa University School of Medicine, Tokyo, Japan

${ }^{49}$ Laboratory for Autoimmune Diseases, RIKEN Center for Integrative Medical

Sciences, Kanagawa, Japan

${ }^{50}$ Department of Internal Medicine, Faculty of Medicine, University of Tsukuba,

Ibaraki, Japan

${ }^{51}$ Department of Statistical Genetics, Osaka University Graduate School of Medicine,

Osaka, Japan

${ }^{52}$ Laboratory of Statistical Immunology, Immunology Frontier Research Center (WPi-

iFReC), Osaka University, Osaka, Japan

${ }^{53}$ Laboratory of Genome Technology, Human Genome Center, Institute of Medical

Science, The University of Tokyo, Tokyo, Japan

${ }^{54}$ Laboratory of Clinical Genome Sequencing, Department of Computational Biology and Medical Sciences, Graduate School of Frontier Sciences, The University of Tokyo, Tokyo, Japan

${ }^{55}$ Division of Cancer Epidemiology and Prevention, Aichi Cancer Center Research Institute, Nagoya, Japan

${ }^{56}$ Department of Epidemiology, Nagoya University Graduate School of Medicine,

Nagoya, Japan

${ }^{57}$ Department of Genomic Function and Diversity, Medical Research Institute, Tokyo

Medical and Dental University, Tokyo, Japan

${ }^{58}$ Department of Pediatrics, University of Cincinnati, Cincinnati, Ohio, USA

${ }^{59}$ US Department of Veterans Affairs Medical Center, Cincinnati, Ohio, USA

${ }^{60}$ Department of Rheumatology and Clinical immunology, Kyoto University Graduate school of Medicine, Kyoto, Japan

${ }^{61}$ Department of Orthopaedic Surgery, Faculty of Medicine, Fukuoka University,

Fukuoka, Japan

${ }^{62}$ State Key Laboratory of Oncogenes and Related Genes, Shanghai Cancer Institute, Renji Hospital, Shanghai Jiao Tong University School of Medicine (SJTUSM),

Shanghai, China

${ }^{63}$ Department of Dermatology, Institute of Dermatology, Huashan Hospital, Fudan

University, Shanghai, China

${ }^{64}$ Clinical Research Center, Shizuoka General Hospital, Shizuoka, Japan

${ }^{65}$ The Department of Applied Genetics, The School of Pharmaceutical Sciences,

University of Shizuoka, Shizuoka, Japan 
Acknowledgements We acknowledged the participants in this study. We appreciate the contribution of Japanese Research Committee on Idiopathic Osteonecrosis of the Femoral Head. We appreciate all contributors to BioBank Japan. Details are included in supplementary material.

Contributors $X Y, K K i m$ and $H S$ contributed equally to this work, and either has the right to list himself first in bibliographical documents. SCB, YC, CT, XZhang, XY, KKim and HS conceived the study design. SCB, YC, XZhang, SY, KKim and CT acquainted the financial support. XY, KKim, HS, CT, YC and SCB wrote the manuscript. XY, KKim $\mathrm{HS}$, EH, XZheng, VL and YW conducted all of the analyses with the help of JBH, LCK, MTW, SP, SE, HS, KT, NO, MK, KI and C Terao. KKim, SYB, LW, LL, RXL, YSheng, MYH, WL, KYoon, MC, HH, MW, YTang, HD, CL, CS, WF, KL, BJK, HSL, SCB, SH, YSakamoto, NSugano, MM, DT, KKarino, TMiyamura, JN, GM, TKuroda, HN, TMiyamoto, TT, YKawaguchi, KA, YTada, KYamaji, MS, TA, AS, TSumida, YOkada, KMatsuda, KMatsuo, YKochi, TSeki, YTanaka, TKubo, RH, TYoshioka, MY, TKabata, YA, YOhta, TO, YN, AK, YY, KOhzono, KYamamoto, KOhmura, TYamamoto and SI generated genetic data. SYB, SJ, YCK, WTC, SSL, SCS, YMK, DY, CHS, YBP, JYC, YP, GYA, JMS, YKL, DJP, WY, THK, SY, BJK, NShen, HSL, XZhang, CT and SCB managed the cohort data. All authors reviewed and approved the manuscript.

Funding This research was supported by General Program $(81872516,81573033$, $81872527,81830019,81421001)$, Young Program $(81803117,82003328)$. Exchange Program (81881340424), and Science Fund for Creative Research Groups (31630021) of National Natural Science Foundation of China (NSFC), Distinguished Young Scholar of Provincial Natural Science Foundation of Anhui (1808085J08), National Program on Key Basic Research Project of China (973 Program) (2014CB541901), China National Key R\&D Program (2016YFC0906100), Science Foundation of Ministry of Education of China (213018A), Program for New Century Excellent Talents in University of Ministry of Education of China (NCET-12-0600), The Bio \& Medical Technology Development Program of the National Research Foundation, funded by the Ministry of Science \& ICT of the Republic of Korea (NRF-2017M3A9B4050355 to SCB), Basic Science Research Program through the National Research Foundation of Korea funded by the Ministry of Science, ICT and Future Planning (2015R1C1A1A02036527 and 2017R1E1A1A01076388 to KKim), National BioBank of Korea, the Centers for Disease Control and Prevention, Republic of Korea (KBN-2018-031 to SSL), Center for Genome Science, Korea National Institute of Health, Republic of Korea (4845-301, 3000-3031 to MYH, KYoon and BJK), Japan Agency for Medical Research and Development (AMED) and the BioBank Japan project supported by the Ministry of Education, Culture, Sports, Sciences and Technology of the Japanese Government and AMED under grant numbers (17km0305002 and 18km0605001), Grant of Japan Orthopaedics and Traumatology Research Foundation, Inc, (No. 350 to YSakamoto), RIKEN Junior Research Associate Program (to H.S.), US NIH grants (AI024717, Al130830, Al148276, HG172111 and AR070549 to JBH), US Department of Veterans Affairs (BX001834 to JBH) and Center for Pediatric Genomics Award and CCRF Endowed Scholar Award of Cincinnati Children's Hospital (to MTW).

Competing interests None declared

\section{Patient consent for publication Not required.}

Ethics approval The study protocol was approved by the Institutional Review Board at each participating institute and the meta-analysis study was additionally approved by the Institutional Review Boards at Anhui Medical University, Hanyang University Hospital of Rheumatic Diseases, and RIKEN Center for Medical Sciences.

Provenance and peer review Not commissioned; externally peer reviewed.

Data availability statement All data relevant to the study are included in the article or uploaded as supplementary information. The meta-analysis summary association statistics in the current study are available from the corresponding author on reasonable request.

Supplemental material This content has been supplied by the author(s). It has not been vetted by BMJ Publishing Group Limited (BMJ) and may not have been peer-reviewed. Any opinions or recommendations discussed are solely those of the author(s) and are not endorsed by BMJ. BMJ disclaims all liability and responsibility arising from any reliance placed on the content. Where the content includes any translated material, BMJ does not warrant the accuracy and reliability of the translations (including but not limited to local regulations, clinical guidelines, terminology, drug names and drug dosages), and is not responsible for any error and/or omissions arising from translation and adaptation or otherwise.

Open access This is an open access article distributed in accordance with the Creative Commons Attribution Non Commercial (CC BY-NC 4.0) license, which permits others to distribute, remix, adapt, build upon this work non-commercially, and license their derivative works on different terms, provided the original work is properly cited, appropriate credit is given, any changes made indicated, and the use is non-commercial. See: http://creativecommons.org/licenses/by-nc/4.0/.

\section{ORCID iDs}

Xianyong Yin http://orcid.org/0000-0001-6454-2384

Kwangwoo Kim http://orcid.org/0000-0001-8926-6216
Rui-Xue Leng http://orcid.org/0000-0002-2453-5865

Nobuhiko Sugano http://orcid.org/0000-0003-4035-3989

Dongqing Ye http://orcid.org/0000-0001-6604-9614

Yongfei Wang http://orcid.org/0000-0002-1260-6291

Wanling Yang http://orcid.org/0000-0003-0063-6327

Yukinori Okada http://orcid.org/0000-0002-0311-8472

Leah C Kottyan http://orcid.org/0000-0003-3979-2220

Kazuhiko Yamamoto http://orcid.org/0000-0001-9037-3625

Tae-Hwan Kim http://orcid.org/0000-0002-3542-2276

Nan Shen http://orcid.org/0000-0002-5875-4417

Chikashi Terao http://orcid.org/0000-0002-6452-4095

Sang-Cheol Bae http://orcid.org/0000-0003-4658-1093

\section{REFERENCES}

1 Carter EE, Barr SG, Clarke AE. The global burden of SLE: prevalence, health disparities and socioeconomic impact. Nat Rev Rheumatol 2016;12:605-20.

2 Guerra SG, Vyse TJ, Cunninghame Graham DS. The genetics of lupus: a functional perspective. Arthritis Res Ther 2012;14:211.

3 Gateva V, Sandling JK, Hom G, et al. A large-scale replication study identifies TNIP1, PRDM1, JAZF1, UHRF1BP1 and IL10 as risk loci for systemic lupus erythematosus. Nat Genet 2009;41:1228-33.

4 Han J-W, Zheng H-F, Cui Y, et al. Genome-Wide association study in a Chinese Han population identifies nine new susceptibility loci for systemic lupus erythematosus. Nat Genet 2009:41:1234-7.

5 Cunninghame Graham DS, Morris DL, Bhangale TR, et al. Association of NCF2, IKZF1, IRF8, IFIH1, and TYK2 with systemic lupus erythematosus. PLoS Genet 2011;7:e1002341.

6 Okada Y, Shimane K, Kochi Y, et al. A genome-wide association study identified AFFas a susceptibility locus for systemic lupus eyrthematosus in Japanese. PLoS Genet 2012:8:e1002455.

7 Kim K, Bang S-Y, Lee H-S, et al. The HLA-DRß1 amino acid positions 11-13-26 explain the majority of SLE-MHC associations. Nat Commun 2014;5:5902.

8 Akizuki S, Ishigaki K, Kochi Y, et al. PLD4 is a genetic determinant to systemic lupus erythematosus and involved in murine autoimmune phenotypes. Ann Rheum Dis 2019:78:509-18

9 Morris DL, Sheng Y, Zhang Y, et al. Genome-Wide association meta-analysis in Chinese and European individuals identifies ten new loci associated with systemic lupus erythematosus. Nat Genet 2016;48:940-6.

10 Catalina MD, Owen KA, Labonte AC, et al. The pathogenesis of systemic lupus erythematosus: harnessing big data to understand the molecular basis of lupus. $J$ Autoimmun 2020;110:102359.

11 Sun C, Molineros JE, Looger LL, et al. High-Density genotyping of immune-related loci identifies new SLE risk variants in individuals with Asian ancestry. Nat Genet 2016;48:323-30.

12 Bentham J, Morris DL, Graham DSC, et al. Genetic association analyses implicate aberrant regulation of innate and adaptive immunity genes in the pathogenesis of systemic lupus erythematosus. Nat Genet 2015;47:1457-64.

13 Buniello A, MacArthur JAL, Cerezo M, et al. The NHGRI-EBI GWAS Catalog of published genome-wide association studies, targeted arrays and summary statistics 2019. Nucleic Acids Res 2019:47:D1005-12.

14 Sirugo G, Williams SM, Tishkoff SA. The missing diversity in human genetic studies. Cell 2019:177:26-31.

15 Danchenko N, Satia JA, Anthony MS. Epidemiology of systemic lupus erythematosus: a comparison of worldwide disease burden. Lupus 2006;15:308-18.

16 Morais SA, Isenberg DA. A study of the influence of ethnicity on serology and clinical features in lupus. Lupus 2017;26:17-26.

17 Ishigaki K, Akiyama M, Kanai M, et al. Large-Scale genome-wide association study in a Japanese population identifies novel susceptibility loci across different diseases. Nat Genet 2020;52:669-79.

18 , Auton A, Brooks LD, et al, 1000 Genomes Project Consortium. A global reference for human genetic variation. Nature 2015;526:68-74.

19 Kim Y, Kim B, Han B. The Korean reference genome Project: construction of the reference panel for imputation analysis. Presented at the 61th Annual Meeting of The American Society of Human Genetics; October 6, 2015, Baltimore, MD, 2015

20 Bycroft C, Freeman C, Petkova D, et al. The UK Biobank resource with deep phenotyping and genomic data. Nature 2018:562:203-9.

21 Howie BN, Donnelly P, Marchini J. A flexible and accurate genotype imputation method for the next generation of genome-wide association studies. PLoS Genet 2009;5:e1000529.

22 Das S, Forer L, Schönherr S, et al. Next-Generation genotype imputation service and methods. Nat Genet 2016:48:1284-7.

23 Purcell S, Neale B, Todd-Brown K, et al. PLINK: a tool set for whole-genome association and population-based linkage analyses. Am J Hum Genet 2007:81:559-75

24 Marchini J, Howie B. Genotype imputation for genome-wide association studies. Nat Rev Genet 2010;11:499-511. 


\section{Systemic lupus erythematosus}

25 Cook JP, Mahajan A, Morris AP. Guidance for the utility of linear models in metaanalysis of genetic association studies of binary phenotypes. European Journal of Human Genetics 2017;25:240-5.

26 Willer CJ, Li Y, Abecasis GR. METAL: fast and efficient meta-analysis of genomewide association scans. Bioinformatics 2010;26:2190-1.

27 Yang J, Ferreira T, Morris AP, et al. Conditional and joint multiple-SNP analysis of GWAS summary statistics identifies additional variants influencing complex traits. Nat Genet 2012;44:369-75.

28 Benner C, Spencer CCA, Havulinna AS, et al. FINEMAP: efficient variable selection using summary data from genome-wide association studies. Bioinformatics 2016;32:1493-501.

29 Bulik-Sullivan BK, Loh P-R, Finucane HK, et al. LD Score regression distinguishes confounding from polygenicity in genome-wide association studies. Nat Genet 2015;47:291-5.

30 Finucane HK, Bulik-Sullivan B, Gusev A, et al. Partitioning heritability by functional annotation using genome-wide association summary statistics. Nat Genet 2015;47:1228-35.

31 Terao C, Momozawa Y, Ishigaki K, et al. GWAS of mosaic loss of chromosome $Y$ highlights genetic effects on blood cell differentiation. Nat Commun 2019;10:4719.

32 Bulik-Sullivan B, Finucane HK, Anttila V, et al. An atlas of genetic correlations across human diseases and traits. Nat Genet 2015;47:1236-41.

33 Wen L, Zhu C, Zhu Z, et al. Exome-wide association study identifies four novel loci for systemic lupus erythematosus in Han Chinese population. Ann Rheum Dis 2018;77:417.
34 Zhang Y, Zhang J, Yang J, et al. Meta-Analysis of GWAS on two Chinese populations followed by replication identifies novel genetic variants on the $X$ chromosome associated with systemic lupus erythematosus. Hum Mol Genet 2015;24:274-84.

35 Armstrong DL, Eisenstein M, Zidovetzki R, et al. Systemic lupus erythematosusassociated neutrophil cytosolic factor 2 mutation affects the structure of NADPH oxidase complex. J Biol Chem 2015;290:12595-602.

36 Kim-Howard X, Sun C, Molineros JE, et al. Allelic heterogeneity in NCF2 associated with systemic lupus erythematosus (SLE) susceptibility across four ethnic populations. Hum Mol Genet 2014;23:1656-68.

37 Yang W, Shen N, Ye D-Q, et al. Genome-Wide association study in Asian populations identifies variants in ETS1 and WDFY4 associated with systemic lupus erythematosus. PLoS Genet 2010;6:e1000841.

38 Pasula S, Tessneer KL, Fu Y, et al. Role of systemic lupus erythematosus risk variants with opposing functional effects as a driver of hypomorphic expression of TNIP1 and other genes within a three-dimensional chromatin network. Arthritis Rheumatol 2020;72:780-90.

39 Chen P-W, Luo R, Jian X, et al. The ARF6 GTPase-activating proteins ARAP2 and ACAP1 define distinct endosomal compartments that regulate integrin $\alpha 5 \beta 1$ traffic. J Biol Chem 2014;289:30237-48.

40 Sannigrahi MK, Srinivas CS, Deokate N, et al. The strong propensity of Cadherin-23 for aggregation inhibits cell migration. Mol Oncol 2019;13:1092-109.

41 Morimoto K, Baba Y, Shinohara H, et al. LRRK1 is critical in the regulation of B-cell responses and CARMA1-dependent NF- $\kappa$ B activation. Sci Rep 2016;6:25738.

42 Kwon OC, Lee JS, Ghang B, et al. Predicting eventual development of lupus nephritis at the time of diagnosis of systemic lupus erythematosus. Semin Arthritis Rheum 2018;48:462-6. 